\title{
FEASIBILITY OF SHARPENING LIMITED-ANGLE TOMOGRAPHY BY INCLUDING AN ORTHOGONAL SET OF PROJECTIONS*
}

\author{
Kenneth F. KORAL, Neal H. CLINTHORNE, W. Leslie ROGERS and John W. KEYES, Jr. \\ University of Michigan Medical Center, Ann Arbor, MI 48109, U.S.A.
}

\begin{abstract}
In single photon emission nuclear medicine, computed tomography systems that employ only a limited angular range of projection data suffer from blurring in the depth (or $z$ ) direction. With simulated data, we show that including a second set of projections taken in an orthogonal direction sharpens the image in the $z$ direction. For heart applications, this sharpening improves the sizing of a simulated $2 \mathrm{~cm}$ diameter "hot" infarct by $39 \%$. It also should improve the contrast of "cold" infarcts by eliminating blurring from other planes, but here we have found a complication, in that, with the present approximate algorithm, effects from the lack of symmetry in two-view tomography may overshadow benefits from contrast improvement. Further simulation study is called for.
\end{abstract}

\section{Introduction}

In tomographic nuclear medicine, we wish to obtain a three-dimensional map of the distribution of a radioactive pharmaceutical within the human body. Depending on the isotope, each radioactive decay can produce a positron which, upon annihilation, leads to an approximately opposed pair of high energy gamma rays or it can produce a single gamma ray. This paper is concerned with mapping through the detection of single gamma ray events.

Currently, many single photon emission computed tomography systems are based upon an Anger camera, a large area detector for forming images of the incident gamma ray distribution. There are, however, two general categories of systems based on the Anger camera. The first system employs a parallel hole collimator with channels perpendicular to the camera face and requires that the camera be rotated through $180^{\circ}$ about an axis which is perpendicular to the channels and passes through the patient. A given row of channels then sees only a single slice of the object for all projections and the reconstruction is accomplished slice by slice with many nearly independent data sets.

The second type of system is simpler mechanically and approaches the problem without the breakup into independent slices.

\footnotetext{
* This work was supported by National Institutes of Health grant No. GM28305.
}

With one of a variety of collimators and a fixed camera, the three-dimensional object is viewed from many different angles but over only a limited range, defined by the size of the camera face and the depth of the object element. The entire object volume is then reconstructed at once. All of the systems falling into this second category suffer from degraded resolution in the direction perpendicular to the face of the Anger camera (the $z$ direction) due to the lack of completeness in the angular range of the projections. For volume objects, several studies

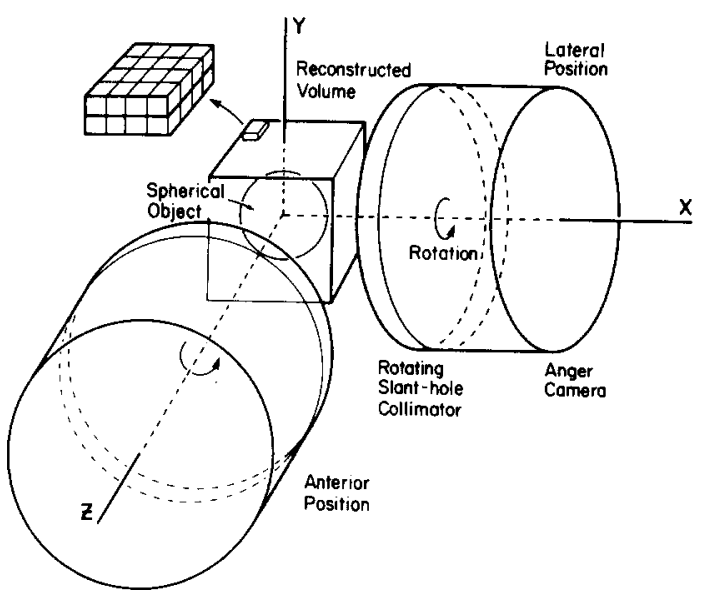

Fig. 1. In two-view tomography, a set of projections is taken with the camera in the anterior position by rotating the slant-hole collimator. Then the camera is rotated $90^{\circ}$ about the $y$ axis to the lateral position and a second set of projections recorded. At the upper left, a small section of the reconstructed volume shows that the elements are cubes. 


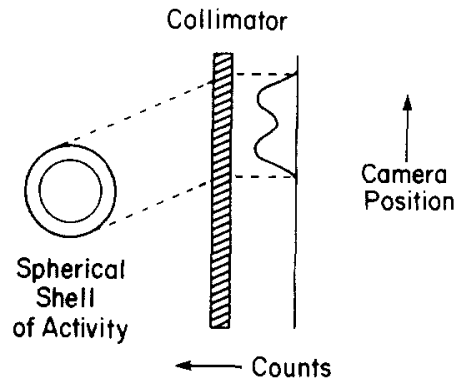

Fig. 2. A two-dimensional section of a rotating slant-hole collimator for one angular position. A plot of detected counts versus camera position is shown for a spherical shell. In three-dimensions, all collimator channels are parallel. As a function of time, the collimator rotates in angular increments about an axis perpendicular to the camera face, and a projection image is taken for each increment.

$[1,2]$ under different conditions and using different assumptions have shown that this degraded resolution leads to images which are elongated in the $z$ direction compared with the object. Phantom studies [3] and canine infarct studies [4] have shown that this degraded resolution causes problems in using the images, particularly when the purpose of the study is to estimate the size of diseased zones.

In this paper, we investigate the feasibility of improving $z$ resolution by rotating the camera $90^{\circ}$ about the $y$ axis (see fig. 1) and taking a second set of tomographic projections. In principle, this measurement is possible with any of the limited-angular-range systems including the seven pinhole collimator [5], the pinhole timecoded aperture [6], the rotating slant hole collimator [7], or the quadrant slant hole collimator [8]. The reconstruction algorithm is easiest to implement for the slant-hole collimator (fig. 2), however, since a cube-shaped image element is the natural choice for this collimator and this element is symmetric with respect to the $90^{\circ}$ rotation. This system, then, will be assumed for the rest of the paper.

\section{Application}

The probable application of two-view limitedangular-range tomography is the imaging of the heart or some other relatively small object. Imaging a large extended organ like the liver calls for transverse section tomography. We will assume a slant angle of $26^{\circ}$ with the normal to the camera and will concentrate on the left ventricle of the heart. Then it is possible that this object could be completely imaged for all rotation angles of the collimator with a 10 inch diameter camera even at the approximately $6 \mathrm{~cm}$ greater depth for the lateral view compared with the anterior view. Recognizing that positioning the patient would be difficult in this case and that it is desirable to image other chambers of the heart if possible, we will take the more conservative approach of assuming a 15 inch diameter camera. For each angle of rotation, a different section of this large camera area is needed to carry out the reconstruction, but it is not necessary to process the entire area.

There are two different methods for looking for infarcted areas of the wall of the left ventricle: (1) thallium imaging in which the healthy tissue takes up the pharmaceutical and the infarct appears as a non-radioactive region; and (2) technetium pyrophosphate imaging in which the infarct itself takes up the pharmaceutical and the normal heart muscle does not. In this paper, the simplest applicable simulations for these two cases are considered. An $8 \mathrm{~cm}$-outer-diameter spherical shell $1 \mathrm{~cm}$ thick simulates a healthy heart for the thallium case and a $2 \mathrm{~cm}$-diameter solid sphere makes up a pyrophosphate-case infarct. For this initial study, effects of gamma-ray attenuation and of camera mispositioning are ignored. Similarly, uptake by other parts of the body is not considered. For an exercise thallium study, this neglect is appropriate, but for a pyrophosphate application, uptake by bone not viewed in all projections could produce a serious complication by making the different projections inconsistent.

As far as the mechanical method for making two views possible, a camera which rotates about an axis passing through the patient would not be necessary if sufficient patient cot movement were available and the camera could be accurately and conveniently positioned and rotated $90^{\circ}$.

\section{Data simulation and reconstruction algorithm}

Data were simulated for the $8 \mathrm{~cm}$-diameter spherical shell with a $128 \times 128$ image digitization implying a $3.4 \mathrm{~mm}$ square detector element and a 
$3.4 \mathrm{~mm}$ cubic image element (see fig. 1). For the $2 \mathrm{~cm}$-diameter solid sphere, it was necessary to increase the digitization to $256 \times 256$ thus lowering the detector and image element dimension to $1.7 \mathrm{~mm}$. Both objects were assumed centered at the origin and a $31 \times 31 \times 37$ element $(x, y, z)$ reconstruction volume was chosen to better see elongation in the $z$ direction when only the anterior view is employed. To compute the simulated projections, a closed form expression for the length of the appropriate ray through the object was used.

A simultaneous-iterative-reconstruction-technique-(SIRT)-type algorithm [9] with an impedance first estimate [10] is employed for both the one- and two-view reconstructions. No empirical background subtraction is called for under our assumptions. The formulation, including an impedance-estimate normalization factor and an iterative-correction relaxation parameter, is outlined below. The subscripts to accomplish fore- and back-projection are suppressed.

First, to accomplish the impedance estimate

$\frac{1}{I^{0}}=\frac{K}{N} \sum_{i=1}^{N} \frac{1}{P_{i}}$

where $I^{0}$ is the zeroth-iteration image element, $P_{i}$ is the $i$ th projection element, $N$ is the total number of projections ( 12 for 1 view, 24 for 2 views), and $K$ is a normalization factor the suggested value of which is $T$, the greatest object thickness in image elements. The use of multiplication by $K / N$ is introduced here to provide good estimation for both a point and a uniform, symmetric volume object (the value of $K$ changes from 1 to say 12 between these two and brings the estimate into quantitative agreement). A priori information about the object is being invoked. The case for zero projection element is handled separately. Then, to accomplish the $\rho$ th iteration

$E_{i}^{\rho-1}=P_{i}-R_{i}^{\rho-1}$,

where $E_{i}^{\rho-1}$ is the $i$ th element of the error projection and $R_{i}^{p^{-1}}$ is the $i$ th ray sum for the current image and

$I^{\rho}=I^{\rho-1}+\left(r \frac{1}{N_{\mathrm{p}}} \sum_{i=1}^{N} E_{i}^{\rho-1} / N\right)$,

where $N_{\mathrm{p}}$ is the number of elements in a dimension of the reconstruction volume ( 37 here) and $r$ is a relaxation parameter with a suggested value between 1 and $N_{\mathrm{p}} / T$. The later value, always greater than 1 , tends to speed up convergence.

The CPU time for 6 iterations of the algorithm for 2 views or 24 projections was about $36 \mathrm{~s}$ on an Amdahl V/8 computer at a cost, including tape handling, of about $\$ 6.00$.

\section{Results and discussion}

For the spherical shell, a comparison of a two-view reconstruction with the conventional anterior-position one-view reconstruction is shown in fig. 3. The $y-z$ plane itself and two planes parallel to it at the distances of 1.7 and $3.1 \mathrm{~cm}$ are pictured. Across the bottom of the figure is the true object in those planes. It is seen that the one-view image does, indeed, have a $z$-axis elongation which is corrected in the twoview image.

These reconstructions were done with $K=12$ and $r=3.08$. The images shown, after one iteration, are quite non-uniform, the effect of which was decreased for the photos by compressing the upper half of the linear scale for one view and the upper quarter of the scale for two views so the object outline could be seen. The one view reconstruction was actually oscillatory for $r=$ 3.08 and a reduction of $r$ to 1 was required for smoother convergence. Qualitatively, the general outlines of the image remain the same with further iterations or different relaxation parameters, although too large a value of $r$ (like 6.16) causes divergence for the two-view case. In the $y-z$ plane, the uniformity of the two-view image continues to be better than that of the one-view image with more iterations.

Characteristics of the uniformity of the twoview reconstruction for the $x-y$ plane are shown in fig. 4. The values are obtained from the seven pinhole GRAPH program which looks for the maximum values along rays emanating from the shell center and spaced every $6^{\circ}$. The strength results have been normalized so that the average over angle equals 100 . The $0^{\circ}$ position is defined as that of the lateral view direction $(x)$ and the $90^{\circ}$ position is that below the plane of camera rotation $(-y)$. After one iteration, two polar caps at $90^{\circ}$ and $270^{\circ}$ are visible and the standard deviation is \pm 19.8 . After six iterations, the caps 


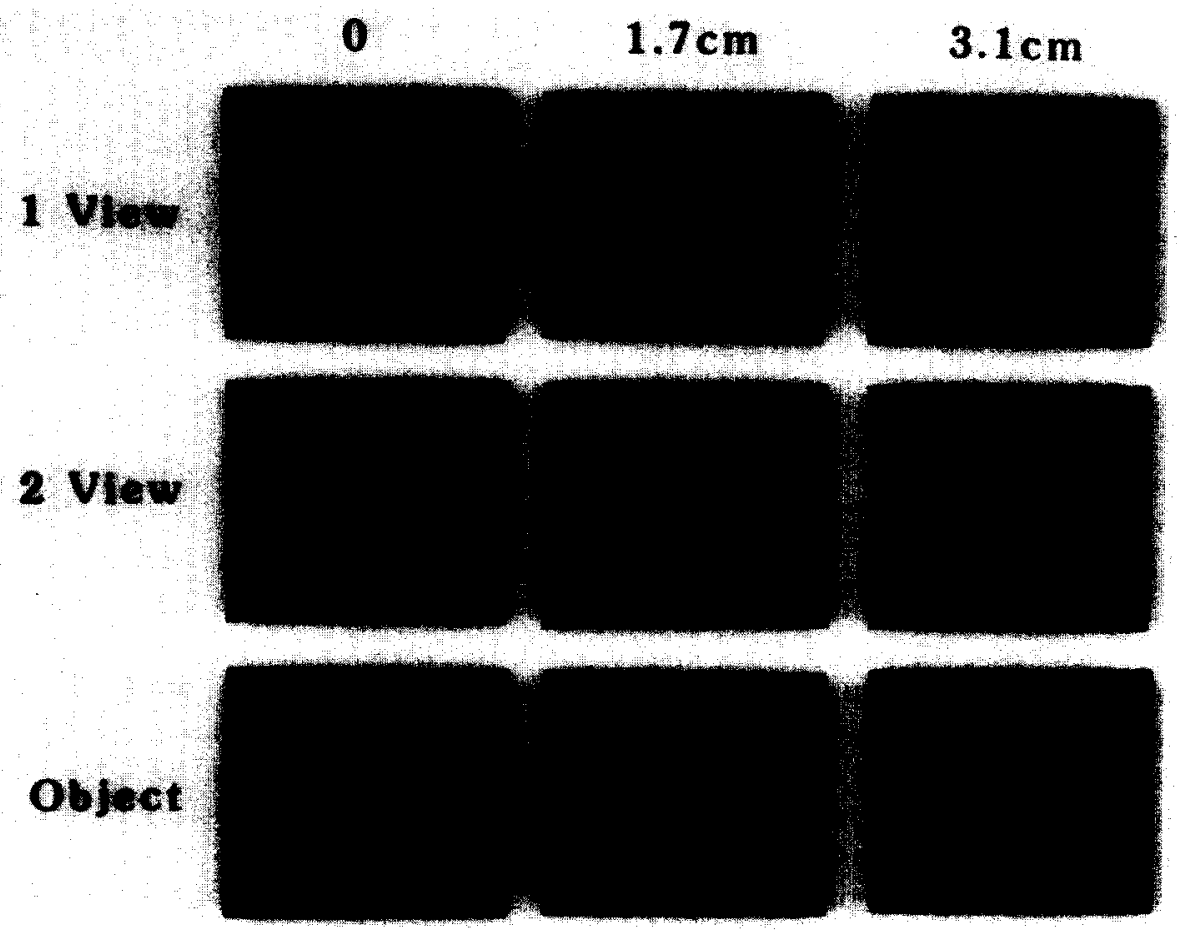

Fig. 3. Results for a $8 \mathrm{~cm}$-outer-diameter, $1 \mathrm{~cm}$-thick spherical shell modeling the left ventricle of the heart. The $y-z$ plane (see fig. 2) and two planes parallel to it at the distances given are shown. Slice thickness is $3.7 \mathrm{~mm}$. The rows form the results from reconstruction with one view, with two views, and from slicing the object itself. The one-view image is elongated toward and away from the camera; the two-view image is faithful in size to the object.

are essentially gone and the standard deviation has decreased to \pm 7.5 . Out to 16 iterations, there is no further significant change in the uniformity. The fact that the image does not converge to a perfectly uniform shell, even with noiseless data,

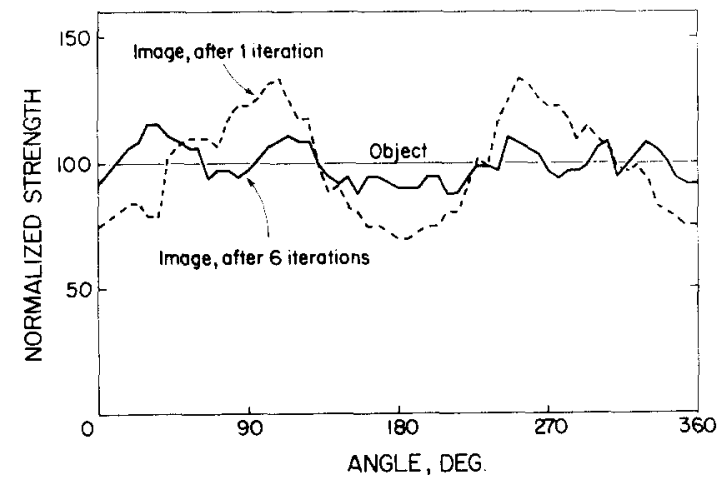

Fig. 4. Uniformity of reconstruction of central plane through spherical shell from simulated two-view data. Images were previously smoothed once to make curves easier to follow. There is an improvement between one and six iterations, but the reconstruction does not converge to the flat trace of the object itself. is attributed to lack of interpolation in the reconstruction algorithm. Simulation studies with perfectly consistent data (generated including the approximations of the algorithm) should help substantiate the cause of the problem. The image non-uniformity is a serious concern for thallium studies since it can mimic an infarct.

It should be noted that the one-view reconstruction, with the same approximations for the same plane after one iteration with $K=37$ and $r=1$, has a standard deviation of only \pm 2.1 . This finding leads to the conjecture that, in the case of real data and the present algorithm, inconsistencies from noise may also be aggravated by the asymmetry of two-view reconstruction leading to further deleterious loss of uniformity. If this is so, then improvement in the accuracy of the algorithm may be called for even if it means longer running times.

For the solid $2 \mathrm{~cm}$ sphere, an elongation similar to that of fig. 3 is found when reconstructing with the data from only one view, whether the anterior or lateral. The elongation is eli- 
minated by using two views. The true volume is $4.19 \mathrm{~cm}^{3}$. The one-view reconstruction leads to a volume determination of $5.96 \mathrm{~cm}^{3}$ which is $42 \%$ high. The two-view reconstruction leads to a determination of $4.08 \mathrm{~cm}^{3}$ which is $3 \%$ low, a marked improvement. These values are based upon sums of plane by plane volume determinations as given by a commercial secondderivative edge-detection program.

In conclusion, these results suggest that in comparison with reconstruction from one view, reconstruction from two-views can lead to more faithful images with available algorithms in reasonable times, yielding possibly an important improvement, especially in the sizing of heart infarcts. Further simulation studies are necessary to explore the limitations of this technique for heart imaging.

\section{References}

[1] J.D. Colsher, Comp. Graph. and Image Proc. 6 (1977) 513.
[2] M.Y. Chiu, H.H. Barrett, R.G. Simpson, C. Chow, J.W Arendt and G.R. Gindi, J. Opt. Soc. Am. 69 (1979) 1323.

[3] D.L. Williams, J.L. Ritchie, G.D. Harp, J.H. Caldwell and G.W. Hamilton, J. Nucl. Med. 21 (1980) 821.

[4] E.M. Stokely, D.M. Tipton, L.M. Buja, S.E. Lewis, M.D. DeVous, Sr., F.J. Bonte, R.W. Parkey and J.T. Willerson, J. Nucl. Med. 22 (1981) 55.

[5] R.A. Vogel, D.L. Kirch, M.T. LeFree and P.P. Steele, J. Nucl. Med. 19 (1978) 648.

[6] K.F. Koral and W.L. Rogers, Phys. Med. Biol. 24 (1979) 879.

[7] S.C. Gottschalk, K.A. Smith and R.H. Wake, J. Nucl. Med. (1980) 27 (abst.); Proc. American College of Nuclear Physicians Mtg (March, 1980).

[8] W. Chang, S.L. Lin and R.E. Henkin, Single photon emission computed tomography and other selected computer topics, ed., J.A. Sorenson (Society of Nuclear Medicine, New York, 1980) p. 81.

[9] P. Gilbert, J. Theor. Biol. 36 (1972) 105.

[10] M.T. LeFree, R.A. Vogel, D.L. Kirch and P.P. Steele, J. Nucl. Med. 22 (1981) 48. 